\title{
The Applicability of Electrocardiogram (ECG) As Biometrics in Securing the Communication of Wireless Body Area Network
}

\author{
Sofia Najwa Ramli ${ }^{1}$, Rabiah Ahmad ${ }^{2}$, Mohd Faizal Abdollah ${ }^{3}$ \\ ${ }^{1,2,3}$ Center for Advanced Computing Technology \\ Universiti Teknikal Malaysia Melaka \\ Melaka, Malaysia
}

\begin{abstract}
A recent trend in the field of biometrics is ECGbased, where electrocardiogram (ECG) signals are used as input to the biometric system. Previous work has shown that ECG has a good potential, which can be used alone as a biometric parameter or in combination with some other parameters for greater accuracy, due to its main key properties. This paper presents a study on the applicability of ECG signals in securing Wireless Body Area Network (WBAN) communications. We study the permanence and the distinctiveness properties of ECG signals on 20 random patients. The Independent Component Analysis (ICA) and Fast Fourier Transform (FFT) are applied on the ECG signals obtained from MITBIH Normal Synus Rhythm (nsrdb) and MIT-BIH Arrhythmia (mitdb) public database. The experimental results are presented, which exhibit that ECG signal can be utilized properly to achieve better security performance under the stringent constraints of WBAN sensors. Thus, it is believed that the system can naturally secure the information transmission within WBAN, where other techniques use hardware and software to achieve the same purpose.
\end{abstract}

\section{Introduction}

Inherent in biometric-based security is both the permanence and distinctiveness of every individual. Recently, cardiovascular signals have been studied for biometric purposes using Electrocardiogram (ECG). The idea of identifying subjects using ECG signal has been investigated impressively due to some key benefits. The main key benefit is the robustness against fabricated credentials as it is hard to steal and impossible to mimic it. Additionally, ECG signal can give the liveliness proof, suggesting that potential application will have a way to reassure that the subject who is offering the biometric is indeed the one who carrying it. ECG also provides additional information related to psychological states and status, which may be of interest for certain applications, as in this paper, for health monitoring in Wireless Body Area Network (WBAN) system.

A WBAN is a network of wireless medical sensors, deployed in, on or around a human body, for enabling ubiquitous, individualized, and real-time health management [1]. Fig. 1 illustrates the position of WBAN in the realm of wireless networks. As the sensors are intentionally implemented to collect physiological signal from human's body; securing the communication over wireless network is very critical. Sensitive medical information must be protected from unauthorized use of personal advantage and fraudulent act that might be harmful to a patient's life. Therefore, it is believed that the collected signal can uniquely represent the dedicated individual and naturally secure the information transmission within WBAN, where other techniques use hardware and software to achieve the same purpose.

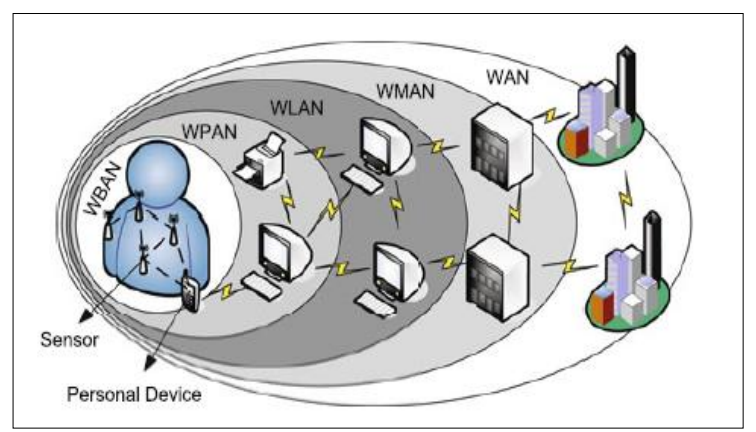

Figure 1. A WBAN in the realm of wireless networks

However, there are several challenges that need to be considered before the ECG biometric can be widely deployed in WBAN. Firstly is to optimize the use of the resources in order to satisfy the stringent constraint of biomedical sensors in terms of power consumption, lightweight, memory space and cost. Secondly, the efficiency of the security system since it is important to have secure sensor networks due to the requirements of medical data privacy, confidentiality, authentication and integrity. These are by far the obstacles to move the technology forward.

\section{The fundamental of ECG}

Electrocardiography is fundamental part of cardiovascular assessment. The contraction and relaxation of cardiac muscle is resulted from the 
depolarization and repolarization of myocardial cells [2]. These electrical changes are recorded via electrodes placed on the limbs and chest wall and are transcribed to a graph paper to produce an ECG. A normal ECG trace consists of a $\mathrm{P}$ wave, a QRS complex and a $\mathrm{T}$ wave. Fig. 2 shows the characteristic of ECG waveform. The P wave is the electrical signature of the current that causes atrial contraction, the QRS complex corresponds to the current that causes contraction of the left and right ventricles, and the $\mathrm{T}$ wave represents the repolarization of the ventricles.

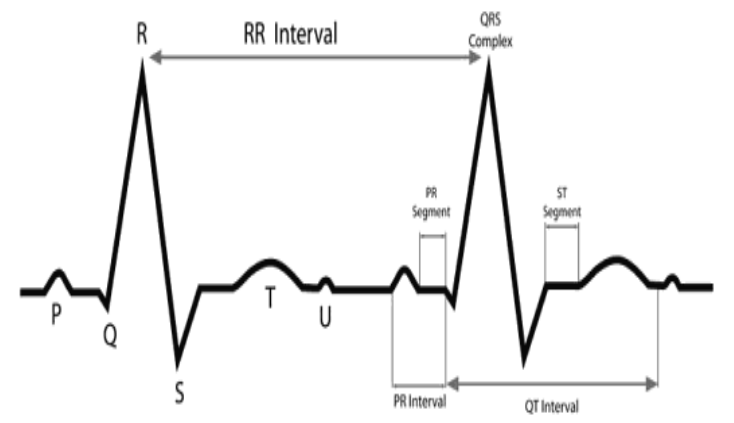

Figure 2. The schematic of ECG waves, segment and intervals [3]

The PQ segment corresponds to electrical impulses transmitted through the S-A node, bundle of His and its branches, and the Purkinje fibers is usually isoelectric. The PQ interval expresses the time elapsed from atrial depolarization to the onset of ventricular depolarization. The ST-T interval coincides with the slow and rapid repolarization of ventricular muscle. The QT interval corresponds to the duration of the ventricular action potential and repolarization. Then TP interval is the period for which atria and ventricles are in diastole. The RR interval represents one cardiac cycle and is used to calculate heart rate.

The QRS complex is the most characteristic waveform of the signal with higher amplitudes. The $\mathrm{R}$ peaks have the largest amplitudes among all the waves making them easiest to detect. However, QRS detection is difficult. It is not only because of the physiological variability of the QRS complex, but also because of the various types of noise that can be present in the ECG signal [4][5]. The contaminated noise sources include muscle noise, artefacts due to electrode motion, power-line interference, baseline wander, and T-waves with high frequency characteristics similar to QRS complex. In order to avoid erroneous conclusions, pre-processing of each ECG signals is very necessary.

\section{ECG for biometrics}

Biometric is a technique commonly known as automatic identification or verification of an individual by his or her physiological and behavioral characteristics [6]. In the other words, Odinaka et al. interpreted that this technique typically operates in either the identification or authentication mode in an identity recognition system [7]. In the identification mode, the system outputs the identity of an individual using the input data. In the authentication mode, the system accepts or rejects a claimed identity associated with input data. If the claimed data is wrongly rejected, the system is said to have experienced a false rejection error. When the claimed identity is wrongly accepted, a false acceptance error occurs.

\subsection{ECG lead placement}

Generally, a standard 12-leads ECG is used for diagnostic test to help identify pathological conditions. It provides a more complete view of the heart's electrical activity and can be used to assess left ventricular function more effectively. Patients with conditions that affect the heart's electrical system may also benefit from 12-lead ECG [8]. 12lead ECG records the heart's electrical activity using a series of electrodes on the body. The 12 leads include three bipolar limb leads, three unipolar augmented limb leads and six unipolar precordial or chest leads.

Generally based on ECG biometrics, all the studies that extract feature from heart's electrical activity are built on one-channel, two-channel, threechannel, or 12-lead channel ECG signals. These are by far the most common lead configurations employed in clinical practice. Among them, most studies generally have employed single-channel ECG signals in biometrics. Many of the single-channel studies use the data from one of the channels of a standard 12-lead ECG recording [7]. However, there are single channel studies that use nonstandard electrodes placement techniques; ECG signals obtained from the pads of individual's thumb [9], ECG signals obtained from palm [10] or ECG signals obtained from electrodes placed bilaterally across the lower rib cage [11]. Furthermore, Biel et al. showed that a signal channel contains sufficient information of feature extraction to support biometrics system [12].

In WBAN system, electrode placement depends on the application itself. Any electrode's sensor not in contact with the subject cannot measure physiological signals from the subject. We assume that only legitimate sensors are in contact with the body. Therefore, any sensor that intends to collect physiological data from the body needs to be authenticated to prove as it represents the dedicated 
body signals. In other words, when two sensors in a WBAN desire a secured communication, the ECG features need to be extracted from both sensors and compared with each other. But, the process must satisfy the challenges explained in section I.

\subsection{ECG as features}

In general, based on the features that are extracted from ECG signals, some researchers categorize ECG biometric methods as either fiducial-based, non fiducial-based or a hybrid [7][13]. The former describes approaches based on fiducial points found in signal by extracting the temporal, amplitude, area, angle, or dynamic (across heart beats) features from the actual points located on the ECG trace. The features include but are not limited to the amplitudes of the $\mathrm{P}, \mathrm{R}$, and $\mathrm{T}$ waves, the temporal distance between wave boundaries (onset and offset of the P, $\mathrm{Q}, \mathrm{R}, \mathrm{S}$, and $\mathrm{T}$ waves), the area of the waves, and slope information. For fiducial-based feature, the interpulse interval (IPI) and RR interval of ECG dynamic are by far the most common feature used to secure communications within WBAN [14][15][16]. They are concerned with the limited resource issues of WBAN sensors. To overcome the problem, the groups proposed a solution by generating symmetric key for encryption and decryption through error correction code that took advantage of ECG features as unique feature.

The non fiducial-based methods do not use the actual points of ECG trace for generating the feature set. Instead, features like wavelet coefficients and autocorrelation coefficients are utilized. Some of the algorithms use one or more of the actual points on the ECG trace for heartbeat segmentation, while others do not use those points at all, but segment the ECG recording into windows that may be overlapping or non-overlapping. Then, they extract features from those windows. Honggang Wang et al. utilized non fiducial-based features to focus on eliminating key distribution issues and overhead caused by time synchronization when using ECG dynamic as features [17][18] in WBAN. They claimed that using dynamic nature of human body would result low chances of physiological signal features being exactly identical. Instead, the authors in [1] was the first to introduce ECG signal processing in frequency domain to extract non fiducial features. Partially fiducial and non-fiducial method, there are a few algorithms that combine nonfiducial features with fiducial features to create the feature set. So far, there is none using hybrid methods in securing WBAN.

\section{Preliminaries}

In this section, we present some preliminaries regarding the use of ECG signal as a feature for securing WBAN.

\subsection{Inter-individual independency of ECG}

In order to satisfy the requirements for universality and permanence, it is necessary to examine the uniqueness of ECG signals for every subject. In this paper, we studied the distinctiveness of each ECG signals using Independent Component Analysis (ICA). B. Kumar et al. claimed that the use of ICA on ECG signal has been justified [19]. In this work, the purpose of using ICA is to extract the independent component of each signal. Such components may represent every signal individually and thus, it can help to prove that these signals are independent between each other.

ICA is data driven computation technique that originally proposed to solve the blind source separation (BSS) problem which aims to separate mixture signals into a set of underlying independent sources without any prior information about either the sources or the mixing parameters [20]. The ICA seeks to estimate the sources by assuming that the output are dominated by a set of hidden sources which are statistically independent of each other and contribute to each output. ICA assumes that underlying sources are statistically independent and non-Gaussian. ICA can be represented by an ICA mixing model given by (1)

$$
X(t)=A_{m x n} S=A_{m x n}\left[\begin{array}{c}
s_{1} \\
s_{2} \\
\vdots \\
s_{n}
\end{array}\right]
$$

The starting point is by given a hypothesis that the recorded ECG signals are a mixed components, $\mathrm{X}(\mathrm{t})$ composed by $\mathrm{x}_{1}(\mathrm{t}), \mathrm{x}_{2}(\mathrm{t}), \mathrm{x}_{3}(\mathrm{t}), \ldots, \mathrm{x}_{\mathrm{m}}(\mathrm{t})$ with $\mathrm{x}(\mathrm{t})$ are the amplitudes, $m=$ number of signals correspond to number of patients and t, the time index. Each of these signals is a weighted sum of signal emitted by unknown sources, which are denoted by $\mathrm{s}_{1}(\mathrm{t}), \mathrm{s}_{2}(\mathrm{t}), \mathrm{s}_{3}(\mathrm{t}), \ldots, \mathrm{s}_{\mathrm{n}}(\mathrm{t})$ with $\mathrm{n}=$ number of independent components.

Each row vector in $\mathrm{X}(\mathrm{t})$ is a linear mixture of hidden and independent process. Each process forms a row vector in $\mathrm{S}$. Each row in $\mathrm{S}$ is an independent component, IC. A is an unknown mxn matrix of full rank called the mixing matrix. The main aim of ICA is then to estimate $\mathrm{S}$ and the mixing matrix, $\mathrm{A}$ from only the observed vector, $\mathrm{X}$. 


\subsection{Feature Generation of ECG}

Bao et al. has claimed that the most expensive operation in terms of energy is the communication operation and the cost of computation is so small compared to communication [14]. Hence, it is important to keep the cost of communication to the minimum. In previous researches, a lot of researchers tried to overcome the above issues by using dynamic features of ECG signals as symmetric key for encryption and decryption to secure WBAN. However, the captured features have varied slightly when measured at the different part of the body. Therefore, they needed to employ time synchronization process to record the timestamp upon arrival of the synchronization signals, as this process will surely increase the cost of communication operation.

Later, some other researchers came out with a solution by processing the ECG signals in frequency domain. It is due to the frequency components of ECG signals, at any given time, have statistically similar values as long as they measured on the same body. Besides, the level of synchronization required for measuring the physiological signals at the sensors is not very strict. Hence, the similar values of these frequency components can be utilized as biometrics for communication within WBAN. In this work, we also explored the method of feature generation in frequency domain using Fast Fourier Transform (FFT).

\section{Results}

A total of 10 random patients' records from two different conditions were prepared as an input for both ICA analysis and frequency domain analysis. Table 1 shows the detailed description of the databases. For each patient, two ECG signals (ECG 1 and ECG 2) were taken from each recorded database. For each recorded signal, the first 4 second of signals were analyzed. The reason of using 4 seconds duration data is to include at least one heart beat in each signal.

The MIT-BIH Normal Synus Rhythm (nsrdb) Database contains 18 long-term excerpts of twochannel ambulatory ECG recordings. The recordings were digitized at 128 samples per second per channel with 12 bits resolution. While the MIT-BIH Arrhythmia Database (mitdb) contains 48 thirty minutes excerpts two-channel ambulatory ECG recordings. The recordings were digitized at 360 samples per second per channel with 11 bits resolution.
Table 1. Patient records used in the study

\begin{tabular}{|c|c|c|}
\hline Database & $\begin{array}{c}\text { MIT-BIH } \\
\text { Normal Synus } \\
\text { Rhythm } \\
\text { (nsrdb) }\end{array}$ & $\begin{array}{c}\text { MIT-BIH } \\
\text { Arrhythmia } \\
\text { (mitdb) }\end{array}$ \\
\hline Heart condition & $\begin{array}{c}\text { Normal heart } \\
\text { rhythm }\end{array}$ & Arrhythmia \\
\hline No. of subjects & 10 & 10 \\
\hline $\begin{array}{c}\text { No. of records } \\
\text { per subject }\end{array}$ & 2 & 2 \\
\hline $\begin{array}{c}\text { Length of each } \\
\text { record }\end{array}$ & 4 seconds & 4 seconds \\
\hline $\begin{array}{c}\text { Sampling } \\
\text { frequency }\end{array}$ & $128 \mathrm{~Hz}$ & $360 \mathrm{~Hz}$ \\
\hline
\end{tabular}

\subsection{ICA for ECG independency}

As discussed in previous chapter, ICA was first applied on the MIT-BIH Normal Synus Rhythm (nsrdb) and MIT-BIH Arrhythmia (mitdb) databases from Physiobank ATM. The 10 random patients' records were prepared as the input of the ICA mixing model. The independent component, ICs and mixing matrix, $A$ was calculated for signal decomposition in each condition. The sample points were whitened and arranged into a $10 \times 512$ data matrix. The ICs and mixing matrix were calculated using FastICA Matlab toolbox. However, it still leaves the ambiguity of the sign for both mixing matrix and ICs. Thus, the mixing matrix, $\mathrm{A}$ is adjusted to have positive peak values to enhance visualization without affecting the ICA model. The sign is represented as in (2).

where

$$
\mathrm{X}=\mathrm{QP}
$$

$$
\begin{aligned}
& \mathrm{Q}=\operatorname{Adiag}\left(\mathrm{SN}_{1}, \mathrm{SN}_{2}, \ldots, \mathrm{SN}_{\mathrm{n}}\right) \\
& \mathrm{P}=\operatorname{diag}\left(\mathrm{SN}_{1}, \mathrm{SN}_{2}, \ldots, \mathrm{SN}_{\mathrm{n}}\right)
\end{aligned}
$$

The $\mathrm{SN}_{\mathrm{i}}(\mathrm{i}=1,2,3 . ., \mathrm{n})$ represent the sign of the dominant peak of every column in the mixing matrix.

The modified mixing matrix, Q, represents the relative ratios in which the independent components exist in the measured signal. In this paper, it will be meaningful, if the mixing ratio of one signal is relatively compared to the other signals. This is realized by normalizing the maximum element of each column of Q to unity as in (5).

where

$$
\mathrm{X}=\mathrm{TC}
$$

$$
\begin{gathered}
\mathrm{T}=\operatorname{Qdiag}\left(\Delta_{1}{ }^{-1}, \Delta_{2}{ }^{-1}, \ldots \Delta_{\mathrm{n}}{ }^{-1}\right) \\
\mathrm{C}=\operatorname{diag}\left(\Delta_{1}, \Delta_{2}, \ldots \Delta_{\mathrm{n}}\right) \mathrm{P}
\end{gathered}
$$


For analysis, the elements of the mixing matrix, $\mathrm{T}$ (after sign and peak modification) has been pointed out in Fig. 3 and Fig. 4 to observe the relative ratios in which the independent components exist in each measured signal.

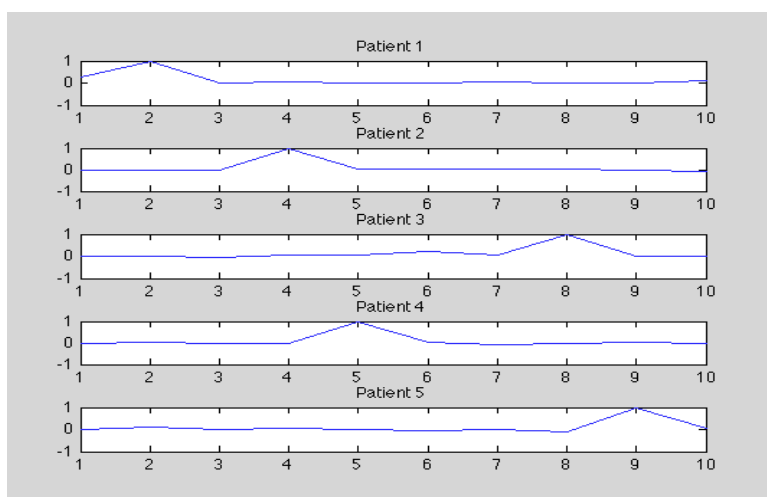

Figure 3. The dominant peak of modified mixing matrix, T for patient 1-5 of ECG 1.

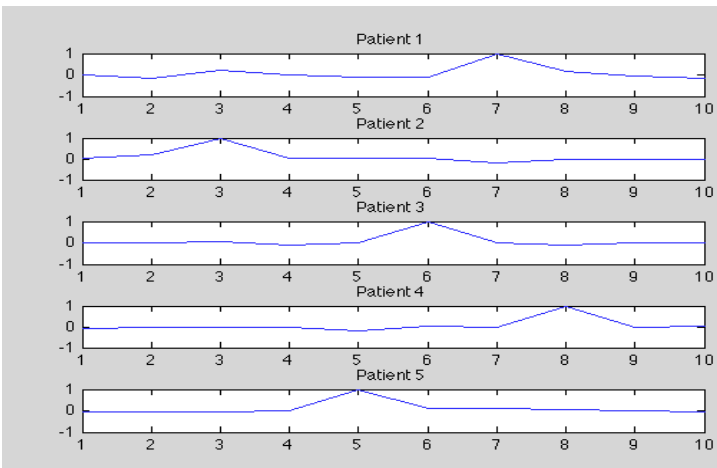

Figure 4. The dominant peak of modified mixing matrix, T for patient 1-5 of ECG 2.

In summary, Table 2 shows that every measured signal of the subject has a strong relationship with each different IC. It is interesting to note that each row of $\mathrm{X}$ is the weighted sum of all rows in $\mathrm{C}$, which represents the ICs. Hence, $X$ is the weighted combination of each column of mixing matrix, $\mathrm{T}$ and all ICs. However, Fig. 2 and 3 indicate that every measured signal is composed strongly from each different IC and approximately zero relative to the other ICs.

For example, patient 1 has a highest relative ratio in which IC 2 (in ECG 1) exists in the measured signal while patient 2 has a highest relative ratio in which IC 4 exists in the measured signal. Therefore, ICA proves that each of the ECG signals is statistically independent to each other. ICA algorithm has extracted different feature from every measured signal and decomposed them into the independent components and mixing ratios without any prior information about either ICs or mixing matrix.
Table 2. The relationship between measured signal, $X$ and $I C, C$

\begin{tabular}{|c|c|c|}
\hline \multirow[t]{2}{*}{ Patients } & \multicolumn{2}{|c|}{$\begin{array}{l}\text { MIT-BIH Normal Synus Rhythm } \\
\text { (nsrdb) }\end{array}$} \\
\hline & ECG 1 & ECG 2 \\
\hline 1 & 2 & 7 \\
\hline 2 & 4 & 3 \\
\hline 3 & 8 & 6 \\
\hline 4 & 5 & 8 \\
\hline 5 & 9 & 5 \\
\hline 6 & 7 & 2 \\
\hline 7 & 10 & 10 \\
\hline 8 & 6 & 4 \\
\hline 9 & 3 & 1 \\
\hline 10 & 1 & 9 \\
\hline \multirow{2}{*}{ Patients } & \multicolumn{2}{|c|}{ MIT-BIH Arrhythmia (mitdb) } \\
\hline & ECG 1 & ECG 2 \\
\hline 1 & 1 & 3 \\
\hline 2 & 3 & 9 \\
\hline 3 & 8 & 10 \\
\hline 4 & 4 & 4 \\
\hline 5 & 6 & 5 \\
\hline 6 & 5 & 2 \\
\hline 7 & 2 & 1 \\
\hline 8 & 9 & 7 \\
\hline 9 & 7 & 8 \\
\hline 10 & 10 & 6 \\
\hline
\end{tabular}

\subsection{ECG feature in frequency domain}

In the previous section, we have proved that the signals are statistically independent to each other. In this section, we try to identify the identical feature of ECG signals for every patient. This feature is needed as biometric of individual during communication in WBAN. In other words, we want to make sure that the number of common features for sensors on the same patient is significantly more than the number of common features for sensors on different patient. Otherwise, other patient can compromise the dedicated patient's WBAN.

Hence, the same data used in ICA was applied in feature generation process. We assumed that the two communicating sensors utilized signals measured from different parts of the body at a given time. The recordings were resampled at $120 \mathrm{~Hz}$ and we took fixed time duration of 4 seconds for every signal. We conducted 512 points FFT of the 4 seconds ECG data 
and extracted the first 256 FFT points because they are symmetric.

Fig. 5 and Fig. 6 indicate local peak values versus peak location index graphs for ECG 1 and ECG 2 obtained from the same individual of MIT-BIH Normal Synus Rhythm (nsrdb) public database. Blue lines represent FFT peaks of ECG 1 and red lines represent FFT peaks of ECG 2. The result has shown that patient 1 has 40 total common peak location index of FFT peak while patient 2 has 51 total common peak location index of FFT peak. Fig. 7 and Fig. 8 show local peak values versus peak location indexes graph for ECG 1 and ECG 2 respectively obtained from different individual. The result has identified that they have 19 total common peak location index of ECG 1 and 25 total common peak location index of ECG 2. The numbers of common peak location index on different patients are only half the numbers of the common peak location index on the same patient.

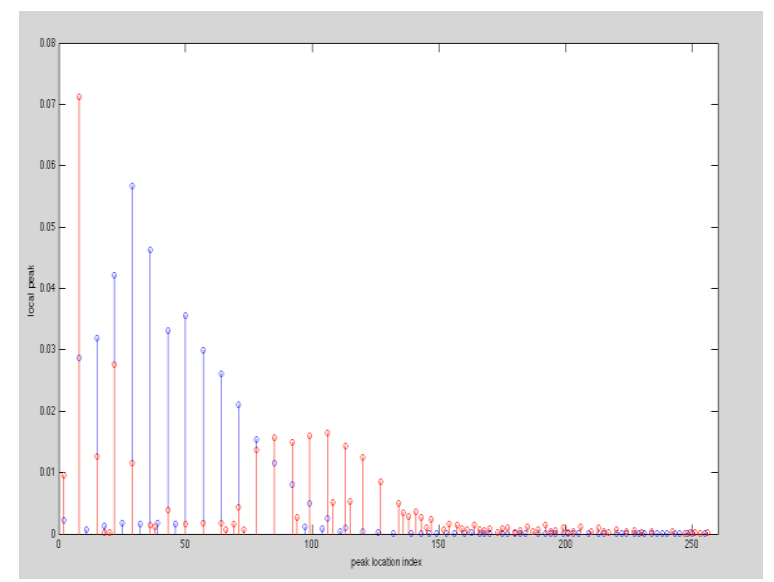

Figure 5. Local peak values versus peak location index for ECG 1 and ECG 2 of patient 1.

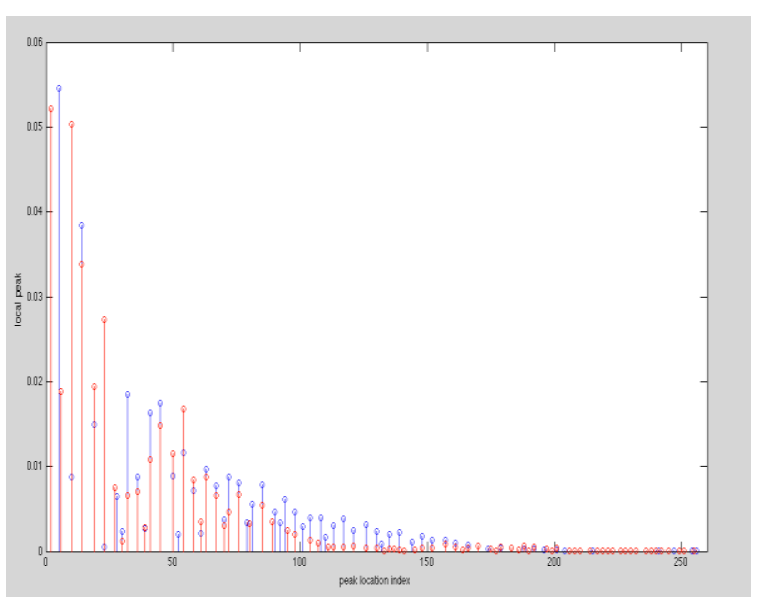

Figure 6. Local peak values versus peak location index for ECG 1 and ECG 2 of patient 2.

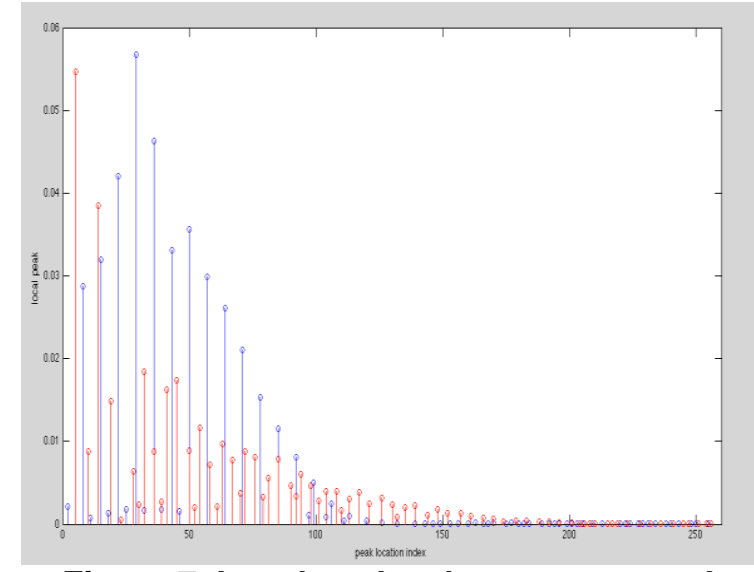

Figure 7. Local peak values versus peak location index of ECG 1 for patient 1 and patient 2.

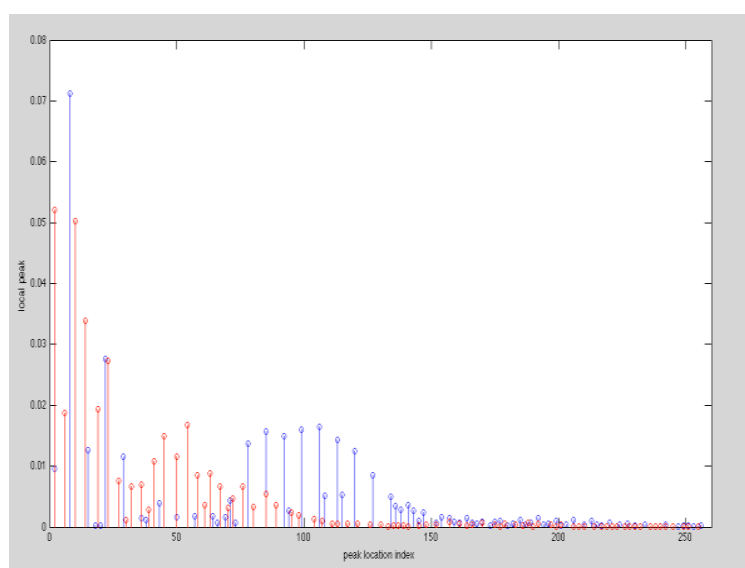

Figure 8. Local peak values versus peak location index of ECG 2 for patient 1 and patient 2.

The same method was applied to MIT-BIH Arrhythmia (mitdb) database. Fig. 9 and Fig. 10 illustrate the common peak locations when the signal collected from the sensors employed on the same individual while Fig. 11 and Fig. 12 demonstrate the common peak locations on different individuals. We found out that patient 1 has 50 total common peak location index on ECG 1 and ECG 2 while patient 2 has 51 total common peak location index on ECG 1 and ECG 2 Besides, we also obtained that patient 1 and patient 2 have only 21 total common peak location index on ECG 1 and 20 total common peak location index on ECG 2.

This exhibit that the number of common feature for sensors on the same subject is significantly higher compared to the number different subject. It is due to the difference in physiological signature of each person at any given time. 


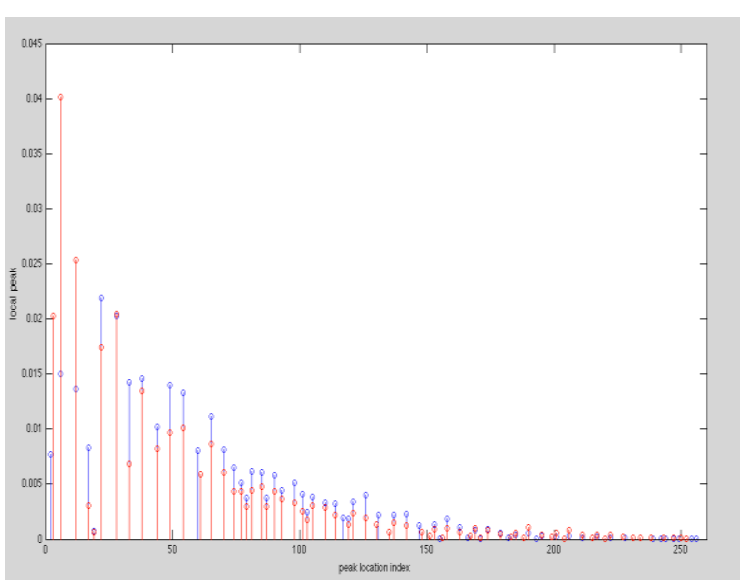

Figure 9. Local peak values versus peak location index for ECG 1 and ECG 2 of patient 1

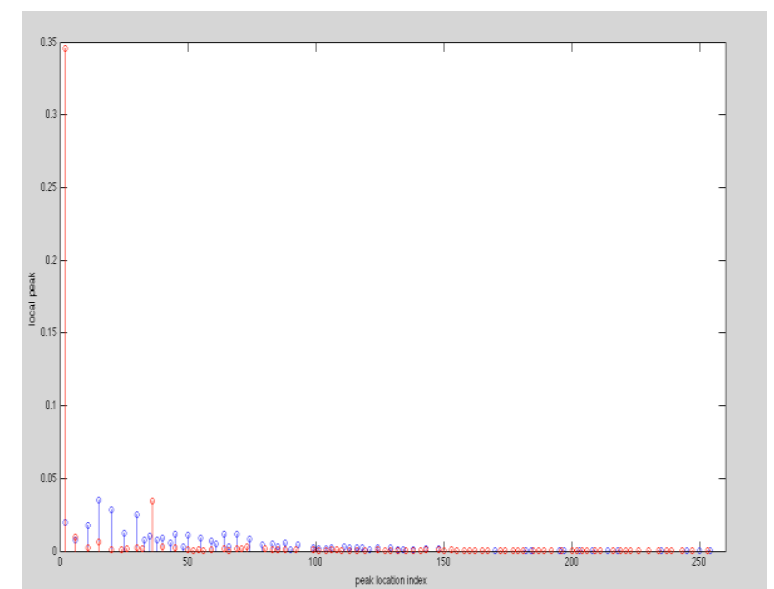

Figure 10. Local peak values versus peak location index for ECG 1 and ECG 2 of patient 2

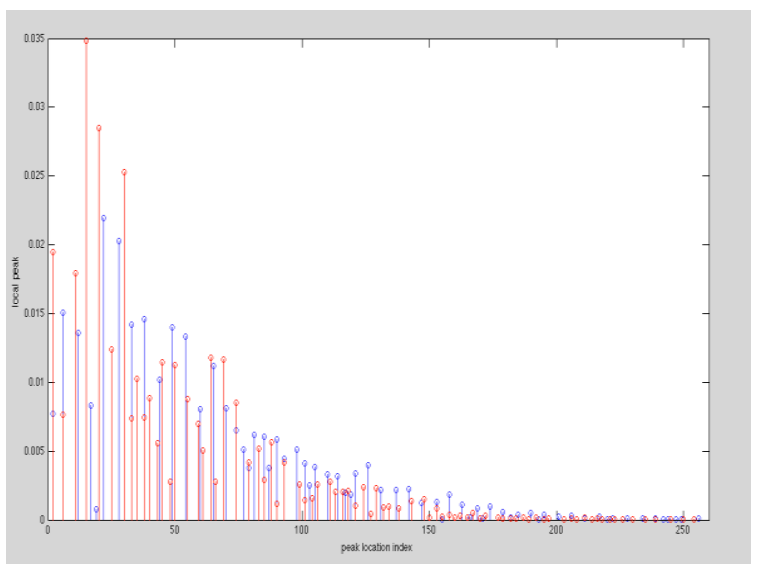

Figure 11. Local peak values versus peak location index of ECG 1 for patient 1 and patient 2

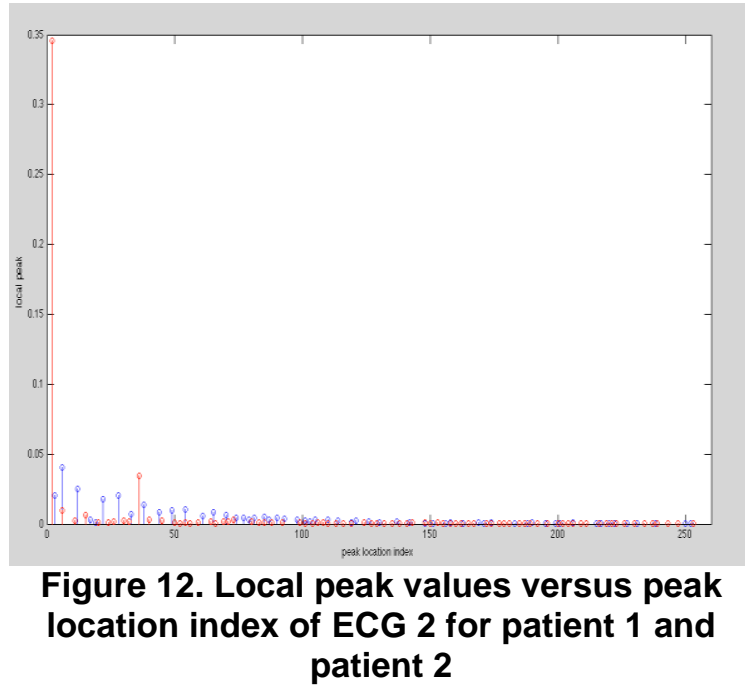

\section{Conclusion}

The inability to replicate ECG signals, is one of the reasons why ECG-based biometric offers an attractive alternative to other conventional biometrics. There are considerable efforts aimed at developing the ECG feature as a biometric modality. But, several important issues remain. These include factors associated with heart rate variability, ECG signal changes over time and privacy concern.

We take a closer look at some of the methodologies that have been proposed by previous researchers. Firstly, we study the distinctiveness of ECG signals using ICA. Secondly, we examine the feature extraction method in frequency domain. The ICA shows that ECG signal of every patient is unique and independent to each other. Precisely, every measured signal is composed strongly from each different independent component and approximately zero relative to the others. In frequency domain analysis, it shows that the number of common feature for sensors on the same subject is significantly higher compared to the number of common feature for sensors on different subject. It is due to the difference in physiological signature of each person at any given time. Therefore, it is believed that the ECG signal can uniquely represent an individual, which is hard to forfeit by suspicious intruders. In other words, patient's records can only be sensed and derived personally by this patient's dedicated WBAN system and will not be mixed with other patients.

Our future work includes the combination of ICA and frequency components of ECG signal to secure the communication under the resource constraints of WBAN sensors. 


\section{Acknowledgement}

The authors would like to thank all the reviewers for their helpful comments. This research is currently supported by the Exploratory Research Grant Scheme (ERGS) funding by the Ministry of Higher Education Malaysia and MyBrain 15 (MyPhD) scholarship program for the academic year of 20112014.

\section{References}

[1] K. K. Venkatasubramanian, A. Banerjee, and S. K. S. Gupta, "PSKA: usable and secure key agreement scheme for body area networks", IEEE transactions on information technology in biomedicine a publication of the IEEE Engineering in Medicine and Biology Society, vol. 14, no. 1, pp. 60-68, 2010.

[2] F. Morris, W. J. Brady, and J. Camm, Eds., $A B C$ of Clinical Electrocardiography, 2nd ed. Blackwell Publishing Ltd, 2008, p. 1.

[3] A. Gacek and W. Pedrycz, Eds., ECG Signal Processing, Classification and Interpretation. Springer, 2012, p. 7

[4] W. J. Pan J., Tompkins, "A Real-Time QRS Detection Algorithm", IEEE Transactions on Biomedical Engineering, vol. BME-32, no. 3, pp. pp.230-236, 1985.

[5] S. K. Singla and A. Sharma, "ECG as Biometric in the Automated World", International Journal of Computer Science and Communication, vol. 1, no. 2, pp. 281-283, 2010.

[6] C. Poon and Y. T. Zhang, "A novel biometrics method to secure wireless body area sensor networks for telemedicine and m-health", Communications Magazine, IEEE, vol. 44, no. 4, pp. 73-81, 2006.

[7] I. Odinaka, S. Member, P. Lai, A. D. Kaplan, J. A. O. Sullivan, E. J. Sirevaag, and J. W. Rohrbaugh, "ECG Biometric Recognition: A Comparative Analysis", IEEE Transactions on Information Forensics and Security, vol. 7, no. 6, pp. 1812-1824, 2012.

[8] J. L. Kowalak and C. Turkington, ECG Interpretation. Lippincot Williams \& Wilkins, 2008, p. 135.

[9] A. D. C. Chan, S. Member, M. M. Hamdy, S. Member, A. Badre, and V. Badee, "Wavelet Distance Measure for Person Identification Using Electrocardiograms", IEEE Transactions on Instrumentation and Measurement, vol. 57, no. 2, pp. 248-253, 2008.

[10] T. D. Shen, W. J. Tompkins, and Y. H. Hu, "Implementation of a one-lead ECG human identification system on a normal population", Journal of Engineering and Computer Innovations, vol. 2, no. January, pp. 12-21, 2011.

[11] I. Odinaka, P.-H. Lai, A. D. Kaplan, J. a. O’Sullivan, E. J. Sirevaag, S. D. Kristjansson, A. K. Sheffield, and J. W. Rohrbaugh, "ECG biometrics: A robust short-time frequency analysis", 2010 IEEE International Workshop on Information Forensics and Security, pp. 1-6, Dec. 2010.

[12] L. Biel, O. Pettersson, L. Philipson, and P. Wide, "ECG Analysis: A New Approach in Human
Identification", Instrumentation and Measurement Technology Conference, 1999. IMTC/99. Proceedings of the 16th IEEE, 1999, vol. 1, pp. 557561.

[13] I. International, W. On, M. Learning, and F. O. R. Signal, "ECG-BASED BIOMETRICS: A REAL TIME CLASSIFICATION APPROACH", IEEE International Workshop on Machine Learning for SIgnal Processing, pp. 1-6, 2012.

[14] S.-D. Bao, L.-F. Shen, and Y.-T. Zhang, "A novel key distribution of body area networks for telemedicine", in 2004 IEEE International Workshop on Biomedical Circuits Systems, 2004, pp. 2-5.

[15] S.-D. Bao, Y.-T. Zhang, and L.-F. Shen, "Physiological signal based entity authentication for body area sensor networks and mobile healthcare systems", $27^{\text {th }}$ Annual International Conference of the IEEE Engineering in Medicine and Biology Society, vol. 3, pp. 2455-8, Jan. 2005.

[16] C. C. Y. Poon, Y.-T. Zhang, and S.-D. Bao, "A novel biometrics method to secure wireless body area sensor networks for telemedicine and m-health", IEEE Communications Magazine, vol. 44, no. 4, pp. 73-81, 2006.

[17] H. Wang, H. Fang, L. Xing, and M. Chen, "An Integrated Biometric-Based Security Framework Using Wavelet-Domain HMM in Wireless Body Area Networks (WBAN)", 2011 IEEE International Conference on Communications (ICC), pp. 1-5, Jun. 2011.

[18] Z. Zhang, H. Wang, A. V Vasilakos, and H. Fang, "ECG-Cryptography and Authentication in Body Area Networks", IEEE Transactions on Information Technology in Biomedicine, vol. 16, no. 6, pp. 10701078, 2012.

[19] C. Ye, M. T. Coimbra, B. V. K. V. Kumar, I. De Telecomunicações, and F. De Ciências, "Investigation of Human Identification using TwoLead Electrocardiogram ( ECG ) Signals", $4^{\text {th }}$ IEEE International Conference on Biometrics: Theory Applications and Systems, pp. 1-8, 2010.

[20] a Hyvärinen and E. Oja, "Independent component analysis: algorithms and applications", Neural networks: the official journal of the International Neural Network Society, vol. 13, no. 4-5, pp. 41130, 2000. 